\title{
Association of perioperative adverse events with subsequent therapy and overall survival in patients with WHO grade III and IV gliomas
}

Lorenz Weber ( $\boldsymbol{\sim}$ lorenz.weber@ksw.ch )

UniversitätsSpital Zürich: UniversitatsSpital Zurich https://orcid.org/0000-0002-6418-9004

Timothy Müller

UniversitätsSpital Zürich: UniversitatsSpital Zurich

Julia Velz

UniversitätsSpital Zürich: UniversitatsSpital Zurich

Flavio Vasella

UniversitätsSpital Zürich: UniversitatsSpital Zurich

Stefanos Voglis

UniversitätsSpital Zürich: UniversitatsSpital Zurich

Luis Padevit

UniversitätsSpital Zürich: UniversitatsSpital Zurich

Dorothee Gramatzki

UniversitätsSpital Zürich: UniversitatsSpital Zurich

Michael Weller

UniversitätsSpital Zürich: UniversitatsSpital Zurich

Luca Regli

UniversitätsSpital Zürich: UniversitatsSpital Zurich

Johannes Sarnthein

UniversitätsSpital Zürich: UniversitatsSpital Zurich

Marian Christoph Neidert

UniversitätsSpital Zürich: UniversitatsSpital Zurich

\section{Research Article}

Keywords: Adverse events, complications, glioblastoma, glioma, treatment delay, neurosurgery

Posted Date: February 11th, 2021

DOl: https://doi.org/10.21203/rs.3.rs-210540/v1 
License: (c) (i) This work is licensed under a Creative Commons Attribution 4.0 International License. Read Full License 


\section{Abstract \\ Purpose}

Maximum-safe-resection followed by chemoradiotherapy as current standard of care for WHO grade III and IV gliomas can be influenced by the occurrence of perioperative adverse events (AE). The aim of this study was to determine the association of $A E$ with the timing and choice of subsequent treatments as well as with overall survival (OS).

\section{Methods}

Prospectively collected data of 283 adult patients undergoing surgery for WHO grade III or IV gliomas at the University Hospital Zurich were analysed. We assessed basic patient characteristics, Karnofsky performance score (KPS), extent of resection (EOR), WHO grade, and we classified AE as well as modality, timing of subsequent treatment (delay, interruption or non-initiation) and OS.

\section{Results}

In 117 patients (41\%), an AE was documented between surgery and 3-months follow-up. There was a significant association of $A E$ with an increased time to initiation of subsequent therapy $(p=0.005)$ and a higher rate of interruption $(p<0.001)$ or non-initiation $(p<0.001)$. AE grades correlated with time to initiation of subsequent therapy $(p=0.038)$. AE were associated with shorter $O S$ in univariate analysis ( $p$ $<0.001)$.

\section{Conclusion}

AE are associated with delayed and/or altered subsequent therapy and worse OS in WHO grade III and IV glioma patients. These data emphasize and quanitfy the importance of safety within the maximum-saferesection concept.

\section{Introduction}

The current standard treatment for WHO grade III and IV gliomas is maximum-safe resection followed by radiotherapy plus/minus concomitant and maintenance alkylating-agent based chemotherapy [1]. Adverse events (AE) are an important and sensitive parameter in measuring management quality of the perioperative period [2]. Importantly, AE occur frequently during and after neurosurgical interventions and are associated with poor functional status [3-5].

$A E$ are considered to affect the course of further therapy and might lead to non-initiation, interruption or delay of subsequent therapy and eventually to worse overall survival (OS) $[5,6]$. However, the association 
of treatment delay of therapy and OS remains controversial. Although the relevance of the exact timing of subsequent therapy remains uncertain, a systemic review [7] and different studies [8-14] seem to agree that a moderate delay of subsequent chemoradiotherapy does not have a detrimental effect on OS, but a delay of more than 42 days after surgery might be associated with worse $0 S$.

Here, we analyzed the association between perioperative AE and both, the timing of subsequent therapy as well as OS.

\section{Methods}

\section{Study design and patient selection}

We prospectively collected data from the patient registry of the Department of Neurosurgery at the University Hospital Zurich [4]. We screened all patients with surgery for anaplastic astrocytoma (WHO grade III) or glioblastoma (WHO grade IV) for eligibility between January 2013 and June 2017. All adult patients with a complete dataset regarding the timing and modality of subsequent therapy and the occurrence of $A E$ were included. Deaths before June 2019 were ascertained and patients were censored at the last date they were stated to be alive.

\section{Treatment}

All included patients underwent initial neurosurgical intervention for WHO grade III or IV glioma. Extent of resection (EOR) varied between biopsy-only, partial resection $<98 \%$ and gross total resection $\geq 98 \%$. The protocol of subsequent therapy during the study period was based on the current EANO guideline [15]. The standard fractionated radiotherapy usually consisted of a dose 60 Gray. First line chemotherapy for glioblastoma during the study period was concomitant temozolomide during radiotherapy followed by 6 cycles of maintenance temozolomide. In case of progression or relapse under the first line treatment with temozolomide, second line chemotherapeutics (mostly CCNU) or bevacizumab were used.

\section{Study variables}

Parameters extracted from the patient registry were age, sex, functional state, EOR, WHO grade, procedure and timing of subsequent therapy, any delay or interruption or non-initiation of the subsequent therapy protocol, the occurrence of $A E$, and OS. The patients' functional state was quantified using the Karnofsky performance score (KPS). A worsened functional state was defined as a decrease of the KPS by $10 \%$ or more 3 months postoperatively compared to the preoperative KPS. EOR was assessed by threedimensional volume measurements of pre- and $<72 \mathrm{~h}$ postopertive MRI using Smartbrush application of Brainlab Elements, Brainlab AG Munich. In case of lacking MRI data, EOR was not further evaluated.

Perioperative AE with its corresponding clinical diagnosis were assessed using the Clavien-Dindo-Grade (CDG) classification and graded accordingly (see Online Resource 1) [16]. 
We defined the variable altered subsequent therapy as the occurrence of either a delay, interruption or non-initiation of subsequent therapy. The time to initiation of subsequent therapy was defined as duration between the intervention and the beginning of subsequent therapy. A delay in subsequent therapy was defined as a duration of more than 42 days until initiation. Any interruptions of therapy were additionally registered and defined as any break in the protocol of subsequent therapy. The non-initiation of subsequent therapy was defined as the absence of subsequent chemo- and radiotherapy.

OS was defined as the time from surgery until death or the last date when the patient was known to be alive.

\section{Statistical methods}

The patient registry is based on FileMaker Pro version 13.0. For statistical analysis and creation of graphs IBM SPSS 24 and MATLAB R2019a were used. A p-value of $\leq 0.05$ was considered significant. Continuous data was analyzed with two-tailed Mann-Whitney U-tests and Student's $t$-tests and categorical data with two-sided Pearson $\chi^{2}$-tests, correlations using Spearman correlation tests, OS using Kaplan-Meier and Log Rank tests and multivariate analysis using Cox's proportional hazard model. Hazard ratios (HR) are given with 95\% confidence intervals (Cl).

\section{Results}

\section{Patient characteristics and outcome data}

Of the 283 included patients, the main clinical characteristics and outcome data stratified for $\mathrm{AE}$ are depicted in Table 1. A statistically significant difference in patient characteristics between patients with $A E$ and patients without AE was seen for age, preoperative KPS, at discharge and at 3 months postoperatively as well as therapy groups. For the outcome data, a statistically significant difference between the subgroups was found for worsened functional state 3 month postoperative, altered subsequent therapy, delay in subsequent therapy, interruption, non-initiation and median OS.

Table 1 Patient characteristics and outcome variables stratified for $A E$. AE, adverse event. EOR, extent of resection.. KPS, Karnofsky performance score. IQR, interquartile range. SD, standard deviation. $C l$, confidence interval. ${ }^{a}$ at least one AE from surgery to 3 months postoperatively. ${ }^{b}$ KPS 3 months postoperatively compared to peroperative KPS. ${ }^{c}$ at least one AE prior to start of subsequent therapy. ${ }^{d}$ Mann-Whitney U-test. ${ }^{e} \chi^{2}$-test. ${ }^{f}$ Student's t-test. ${ }^{g}$ Log Rank test. * statistically significant 
Patient characteristics and outcome variables stratified for $\mathrm{AE}$

\begin{tabular}{|c|c|c|c|c|}
\hline Variables & $\begin{array}{l}\text { Overall }(n= \\
283)\end{array}$ & $\begin{array}{l}\text { no } A E \\
(n=166)\end{array}$ & $\begin{array}{l}A E \\
(n=117)^{a}\end{array}$ & p-Value \\
\hline Median age, years (IQR) & $63(52-72)$ & $61(52-69)$ & $\begin{array}{l}67(54- \\
74.5)\end{array}$ & $0.005^{\mathrm{d}^{*}}$ \\
\hline Female, n (\%) & $95(34)$ & $58(35)$ & $37(32)$ & $0.561^{\mathrm{e}}$ \\
\hline Glioblastoma WHO grade IV, n (\%) & $245(87)$ & $139(84)$ & $106(91)$ & $0.095^{\mathrm{e}}$ \\
\hline \multicolumn{5}{|l|}{ Extent of resection, $\mathrm{n}(\%)$} \\
\hline Biopsy only & $89(31)$ & $47(28)$ & $(36)^{42}$ & $0.176^{\mathrm{e}}$ \\
\hline Partial resection (EOR < 98\%) & $114(40)$ & $72(43)$ & $(36)^{42}$ & $0.207^{e}$ \\
\hline Gross total resection (EOR $\geq 98 \%$ ) & $69(24)$ & $42(25)$ & $(23)^{27}$ & $0.668^{e}$ \\
\hline Unclear extent of resection & $11(4)$ & $5(3)$ & $6(5)$ & $0.364^{\mathrm{e}}$ \\
\hline
\end{tabular}

Therapy groups, n (\%)

Chemoradiotherapy

$174(62)$

(70) 116

$(50)^{58}$

$<0.001^{\mathrm{e}^{*}}$

Chemotherapy

$33(12)$

$22(13)$

(9)

Radiotherapy

$45(16)$

$22(13)$

$(20)^{23}$

No other therapy than best supportive care

$31(11)$

$6(4)$

Median KPS score preoperatively, \% (IQR)

$80(70-90)$

$80(70-90)$

$(21)^{25}$

$80(70-$

90)

Median KPS score at discharge, \% (IQR)

$80(70-90)$

$90(80-90)$

$70(50-$

80)

Median KPS score at 3 months

postoperatively, \% (IQR)

$80(60-90)$

$90(80-90)$

$60(15-$

80)

Worsened functional state ${ }^{\mathrm{b}}, \mathrm{n}(\%)$

$130(46)$

56 (33)

74 (63)

$<0.001^{\text {e* }}$

Mean time to initiation of subsequent

therapy, days (SD)

$32(12)$

$30(8.5)$

$35(17)^{\mathrm{C}}$

$0.005^{f_{*}}$

Altered subsequent therapy, n (\%)

$82(29)$

$23(13)$

59 (50)

$<0.001^{\text {e* }}$

Delay (>42d) in subsequent therapy

28 (11)

12

16

$0.016^{\text {e* }}$ 
$(7.5)$

$\begin{array}{clllll}\text { Interruption } & 29(10) & 5(3.0) & (21)^{24} & <0.001^{\text {e* }} \\ \text { Non-initiation } & 31(11) & 6(3.6) & (21)^{25} & <0.001^{\text {e* }} \\ \text { Median overall survival, months (95\% Cl) } & \begin{array}{l}13(11.3- \\ 14.7)\end{array} & \begin{array}{l}17(14.5- \\ 19.5)\end{array} & \begin{array}{l}9(6.7- \\ 11.3)\end{array} & <0.001^{\text {g* }}\end{array}$

\section{$\mathrm{AE}$ are associated with delay and altered subsequent treatment}

The mean time to initiation of subsequent therapy was significantly higher for patients with $\mathrm{AE}$ prior to therapy $(p=0.005)$ and correlated significantly with the grade of $A E(p=0.038$, Spearman's rho $=0.13$, Fig. 1a). Altered subsequent therapy in general and each of the underlying variables (either delay or interruption or non-initiation of the subsequent therapy) was more frequent in the subgroup of patients with AE (Table 1).

Additionally, there was a significant correlation between the severity of CDG and KPS at discharge from hospital $(p<0.001$, Spearman's rho $=-0.41)$ with a slope of -9.5 KPS points per increment of ClavienDindo-Grade in the linear fit (see Fig. 2).

\section{$\mathrm{AE}$ are associated with worse OS}

OS for patients with AE was shorter $(p<0.001)$ (Table 1, Fig. 1b). However, there was no significant correlation between the CDG of the AE and OS $(p=0.063)$. Altered subsequent therapy and its underlying variables interruption and non-initiation were also associated with a significant decrease in OS (Fig. 1C, $1 d)$. However, the delay itself was not associated with a change in $O S(p=0.113$, Fig. 1d).

A multivariate Cox proportional hazard model was conducted to estimate the association of occurrence of $A E$ and altered subsequent therapy with OS (adjusted for confounders age, sex, WHO grade, preoperative KPS and EOR). There was a trend towards shorter OS in patients with AE with HR $=1.32(\mathrm{Cl}$ $0.96-1.77)$, although this did not reach statistical significance $(p=0.063)$. Altered subsequent therapy had a stronger and significant association with shorter OS (HR = 1.97, Cl 1.44-2.69, $p<0.001)$ (see Table 2).

Table 2 Predictor of overall survival (OS). The putative predicting factors for OS as AE and altered subsequent therapy were analyzed using a Cox proportional hazard model correcting for confounders. $A E$, adverse event. KPS, Karnofsky performance score. SE, standard error. * statistically significant 


\section{Prognostic factors of overall survival}

Variables

Occurrence of AE from surgery to 3 months postoperatively

Altered subsequent therapy

Age

Male sex

Tumor grade (WHO grade)

Preoperative KPS

Extent of resection (reference category biopsy only)
Coefficient $\operatorname{Exp}(B)$
SE $\quad 95 \% \mathrm{Cl} \quad \mathrm{p}$-Value

1.32

1.97

1.04

0.99

2.28

0.99
0.15

$0.99-$

1.77

$0.16 \quad 1.44-$

2.69

$0.01 \quad 1.03-<0.001^{*}$

1.05

$0.14 \quad 0.75-$

1.31

$0.27 \quad 1.33-$

3.89

$<0.01$

$0.98-$

0.99
0.063

$<0.001^{*}$

$0.001 *$

0.949

$0.003 *$

$0.003^{*}$

$\begin{array}{lclll}\text { Partial resection } & 0.65 & 0.16 & \begin{array}{l}0.47- \\ 0.90\end{array} & 0.008^{*} \\ \text { Gross total resection } & 0.50 & 0.19 & \begin{array}{l}0.35- \\ 0.72\end{array} & <0.001^{*} \\ \text { Unclear extent of resection } & 0.79 & 0.36 & \begin{array}{l}0.39- \\ 1.59\end{array} & 0.514\end{array}$

\section{Discussion}

There is only scarce information on the frequency and the exact clinical repercussions of $\mathrm{AE}$ in glioma surgery. The risk of AE in the resection of WHO grade III and IV glioma is relatively high due to the difficult balancing between a goal of maximal tumor resection and new postoperative neurological deficits and other $\mathrm{AE}[5]$. Therefore, knowing the consequence of a perioperative $\mathrm{AE}$ is of utmost importance.

The impact of timing of subsequent therapy on OS is still unclear. Nevertheless different studies indicate that a delay of more than 42 days should be avoided [7-12]. We observed a significantly higher mean time to initiation of subsequent therapy and a corresponding higher rate of delay of more than 42 days for patients with an AE. We also observed that AE with higher CDG scores were associated with a longer time to initiation. These findings emphasize that surgical $A E$ can entrail a suboptimal timing and course of subsequent therapy. In addition, our findings indicate that patients with $A E$ are less likely to receive standard of care therapy; the rate of delay, interruption and non-initiation is significantly higher. 
Regarding OS, patients with AE had significantly decreased median survival on univariate analysis in line with previous studies $[5,17]$. On multivariate analysis, patients with AE had a strongly increased hazard ratio adjusted for possible confounders, although the result was not significant, likely due to the relatively

small size of the study group. The potential detrimental effect of $A E$ on OS can be explained by the direct negative effect of $A E$ on the patient's health status, and furthermore by a higher rate of altered subsequent therapy. The association of non-initiation and interruption of subsequent therapy with decreased OS was clearly shown in this study. However, we did not find any evidence showing that a delay of more than 42 days of subsequent therapy alone is associated with inferior OS.

As a retrospective analysis of prospectively collected data, our study is subjected to the general limitations of retrospective studies. Still, our outcome data was collected prospectively and modified retrospectively only in case of incompleteness, which mimized the risk of information bias.

In conclusion, our results support the hypothesis that $\mathrm{AE}$ reduce the rate of successful and uninterrupted chemoradiotherapy and ultimately limit OS. In the sensitive balancing act between maximal resection and postoperative neurological function, our study quantifies the risk of causing $A E$ and by that a possible impairment of the course of subsequent therapy and OS. Therefore, these findings have implications in risk stratification and quality management of WHO grade III and IV glioma surgery.

\section{Declarations}

Funding: This study did not receive any specific funding.

Conflicts of interest/Competing interests: No conflict of interest or competing interests stated by any author.

Ethics approval: The local ethics committee (Kantonale Ethikkommission Zürich) approved prospective data collection in the patient registry and waived patient consent due to the observational nature of the study (PB-2017-00093). The prospective data collection was registered at clinicaltrials.gov (NCT01628406). The study followed the STROBE (Strengthening the Reporting of Observational Studies in Epidemiology) recommendation for observational studies.

Consent to participate: Not applicable.

Consent for publication: Not applicable.

Availability of data and material: The authors confirm that the data supporting the findings of this study are available within the article and its supplementary materials.

Code availability: Not applicable .

Authors' contributions: Study design: L.W., J.S., M.C.N. Data acquisition: L.W., T.M., J.V., F.V., S.V., L.P., J.S., M.C.N. Statistical analysis: L.W. Interpretation: all authors. Writing of original draft: L.W. Review and 
revision: all authors. Approval of final manuscript: all authors.

\section{References}

1. Weller M, van den Bent M, Tonn JC, Stupp R, Preusser M, Cohen-Jonathan-Moyal E, Henriksson R, Le Rhun E, Balana C, Chinot O, Bendszus M, Reijneveld JC, Dhermain F, French P, Marosi C, Watts C, Oberg I, Pilkington G, Baumert BG, Taphoorn MJB, Hegi M, Westphal M, Reifenberger G, Soffietti R, Wick W (2017) European Association for Neuro-Oncology (EANO) guideline on the diagnosis and treatment of adult astrocytic and oligodendroglial gliomas. Lancet Oncol 18: e315-e329 doi:10.1016/s1470-2045(17)30194-8

2. Birkmeyer JD, Dimick JB, Birkmeyer NJ (2004) Measuring the quality of surgical care: structure, process, or outcomes? Journal of the American College of Surgeons 198: 626-632 doi:10.1016/j.jamcollsurg.2003.11.017

3. Schiavolin S, Broggi M, Acerbi F, Brock S, Schiariti M, Cusin A, Visintini S, Leonardi M, Ferroli P (2015) The Impact of Neurosurgical Complications on Patients' Health Status: A Comparison Between Different Grades of Complications. World Neurosurg 84: 36-40 doi:10.1016/j.wneu.2015.02.008

4. Sarnthein J, Stieglitz L, Clavien PA, Regli L (2016) A Patient Registry to Improve Patient Safety: Recording General Neurosurgery Complications. PloS one 11: e0163154 doi:10.1371/journal.pone.0163154

5. Gulati S, Jakola AS, Nerland US, Weber C, Solheim O (2011) The risk of getting worse: surgically acquired deficits, perioperative complications, and functional outcomes after primary resection of glioblastoma. World Neurosurg 76: 572-579 doi:10.1016/j.wneu.2011.06.014

6. Ening G, Osterheld F, Capper D, Schmieder K, Brenke C (2015) Risk factors for glioblastoma therapy associated complications. Clinical neurology and neurosurgery 134: 55-59 doi:10.1016/j.clineuro.2015.01.006

7. Loureiro LV, Victor Eda S, Callegaro-Filho D, Koch Lde O, Pontes Lde B, Weltman E, Rother ET, Malheiros SM (2016) Minimizing the uncertainties regarding the effects of delaying radiotherapy for Glioblastoma: A systematic review and meta-analysis. Radiotherapy and oncology : journal of the European Society for Therapeutic Radiology and Oncology 118: 1-8 doi:10.1016/j.radonc.2015.11.021

8. Sun MZ, Oh T, Ivan ME, Clark AJ, Safaee M, Sayegh ET, Kaur G, Parsa AT, Bloch O (2015) Survival impact of time to initiation of chemoradiotherapy after resection of newly diagnosed glioblastoma. $J$ Neurosurg 122: 1144-1150 doi:10.3171/2014.9.JNS14193

9. Valduvieco I, Verger E, Bruna J, Caral L, Pujol T, Ribalta T, Boget T, Oleaga L, Pineda E, Graus F (2013) Impact of radiotherapy delay on survival in glioblastoma. Clin Transl Oncol 15: 278-282 doi:10.1007/s12094-012-0916-x

10. Blumenthal DT, Won M, Mehta MP, Gilbert MR, Brown PD, Bokstein F, Brachman DG, Werner-Wasik M, Hunter GK, Valeinis E, Hopkins K, Souhami L, Howard SP, Lieberman FS, Shrieve DC, Wendland MM, Robinson CG, Zhang P, Corn BW (2018) Short delay in initiation of radiotherapy for patients with 
glioblastoma-effect of concurrent chemotherapy: a secondary analysis from the NRG Oncology/Radiation Therapy Oncology Group database. Neuro Oncol 20: 966-974 doi:10.1093/neuonc/noy017

11. Lawrence YR, Blumenthal DT, Matceyevsky D, Kanner AA, Bokstein F, Corn BW (2011) Delayed initiation of radiotherapy for glioblastoma: how important is it to push to the front (or the back) of the line? J Neurooncol 105: 1-7 doi:10.1007/s11060-011-0589-2

12. Press RH, Shafer SL, Jiang R, Buchwald ZS, Abugideiri M, Tian S, Morgan TM, Behera M, Sengupta $S$, Voloschin AD, Olson JJ, Hasan S, Blumenthal DT, Curran WJ, Eaton BR, Shu HG, Zhong J (2020) Optimal timing of chemoradiotherapy after surgical resection of glioblastoma: Stratification by validated prognostic classification. Cancer 126: 3255-3264 doi:10.1002/cncr.32797

13. Irwin C, Hunn M, Purdie G, Hamilton D (2007) Delay in radiotherapy shortens survival in patients with high grade glioma. J Neurooncol 85: 339-343 doi:10.1007/s11060-007-9426-z

14. Spratt DE, Folkert M, Zumsteg ZS, Chan TA, Beal K, Gutin PH, Pentsova E, Yamada Y (2014) Temporal relationship of post-operative radiotherapy with temozolomide and oncologic outcome for glioblastoma. J Neurooncol 116: 357-363 doi:10.1007/s11060-013-1302-4

15. Weller M, van den Bent M, Hopkins K, Tonn JC, Stupp R, Falini A, Cohen-Jonathan-Moyal E, Frappaz D, Henriksson R, Balana C, Chinot O, Ram Z, Reifenberger G, Soffietti R, Wick W (2014) EANO guideline for the diagnosis and treatment of anaplastic gliomas and glioblastoma. Lancet Oncol 15: e395-403 doi:10.1016/s1470-2045(14)70011-7

16. Dindo D, Demartines N, Clavien P-A (2004) Classification of Surgical Complications. Annals of Surgery 240: 205-213 doi:10.1097/01.sla.0000133083.54934.ae

17. Ening G, Osterheld F, Capper D, Schmieder K, Brenke C (2015) Risk factors for glioblastoma therapy associated complications. Clinical neurology and neurosurgery 134: 55-59 doi:10.1016/j.clineuro.2015.01.006

\section{Figures}



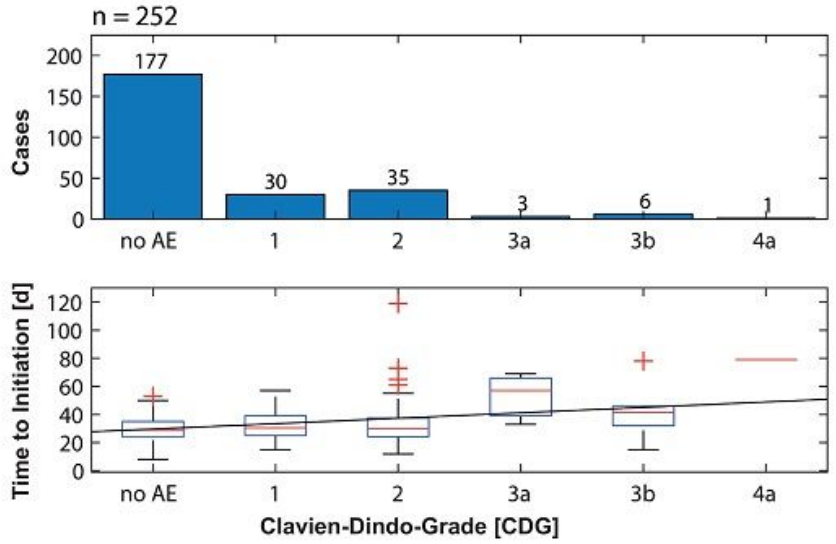

c

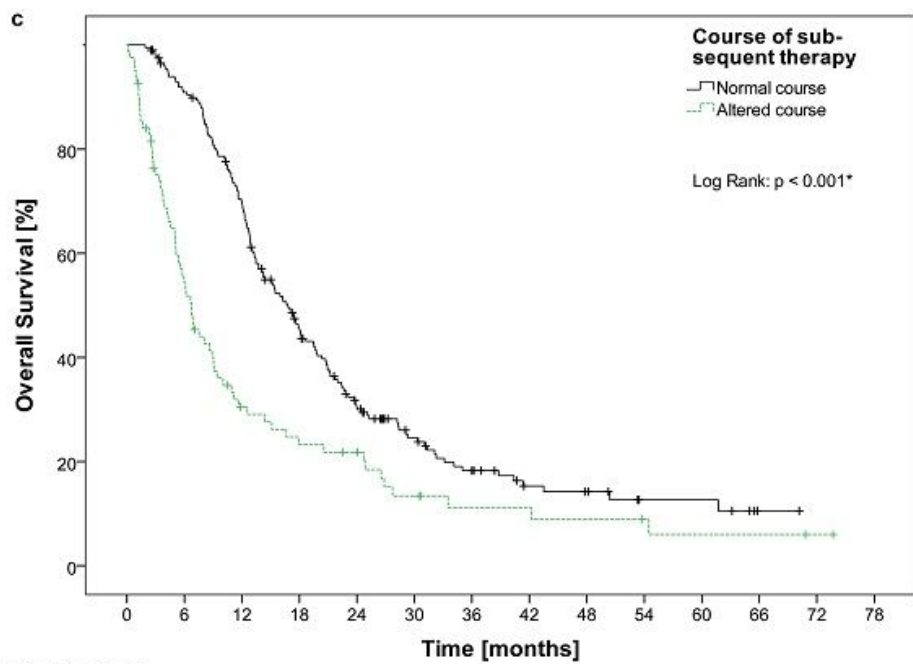

Patients at risk

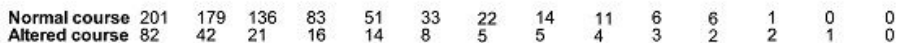

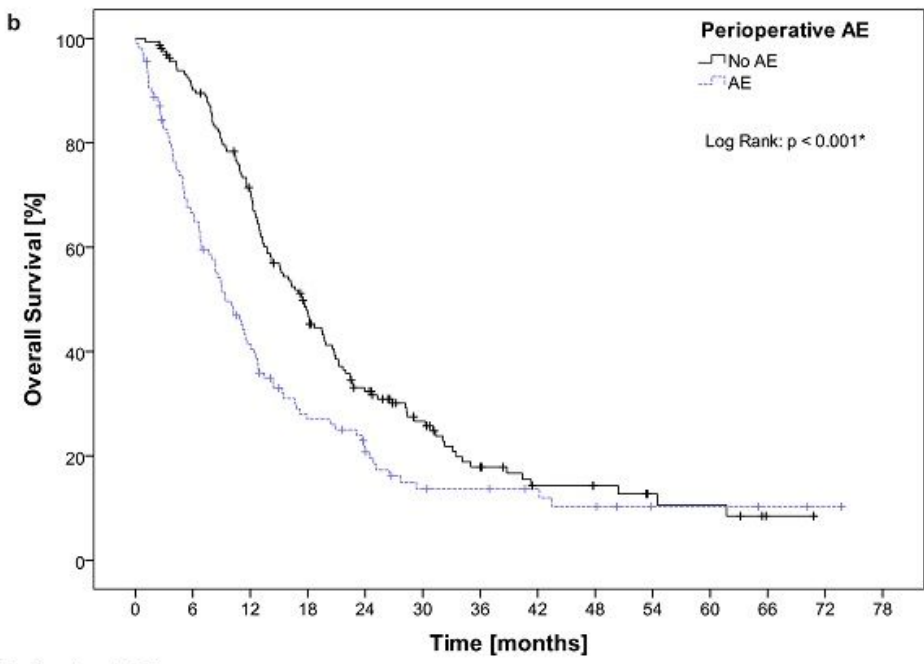

Patients at risk
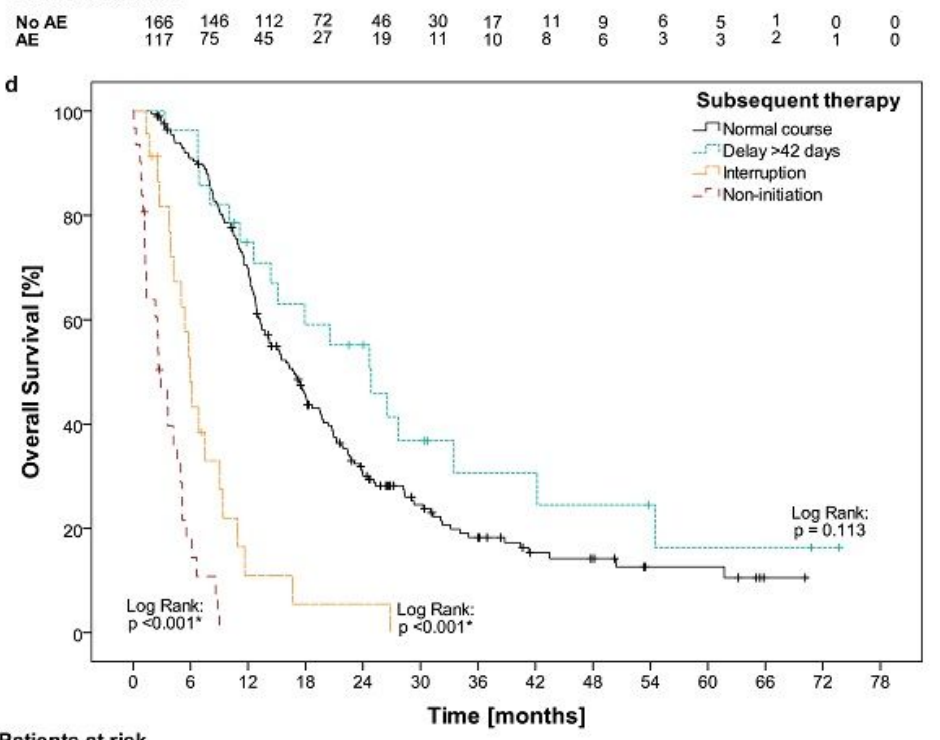

Patients at risk $\begin{array}{lllllllllllllll}\text { Normal course } & 201 & 179 & 136 & 83 & 51 & 33 & 22 & 14 & 11 & 6 & 6 & 1 & 0 & 0 \\ \text { Delay >42d } & 28 & 27 & 19 & 15 & 13 & 8 & 5 & 5 & 4 & 3 & 2 & 2 & 1 & 0 \\ \text { Interruption } & 23 & 10 & 2 & 1 & 1 & 0 & 0 & 0 & 0 & 0 & 0 & 0 & 0 & 0 \\ \text { Non-initiation } & 31 & 5 & 0 & 0 & 0 & 0 & 0 & 0 & 0 & 0 & 0 & 0 & 0 & 0\end{array}$

Figure 1

CDG grade, KPS and subsequent therapy. a: Of all 252 cases with any subsequent therapy, in 75 cases an $A E$ prior to beginning of subsequent therapy was noted. The time to initiation and the CDG grade were correlated with Spearman's rho=0.13 $(p=0.038)$. The linear fit has a slope of 3.9 days per increment of CDG. $b$ : The occurrence of AE until 3 months postoperatively is associated with a significantly lower OS in Log Rank test $(p<0.001) c$ : The occurrence of altered subsequent therapy is associated with a significantly lower OS in Log Rank test $(p<0.001)$. $d$ : The subgroups with interruption or non-initiation of subsequent therapy had both a significant decreased OS $(p<0.001)$. The subgroup with delay showed no significant association with OS $(p=0.113)$ 


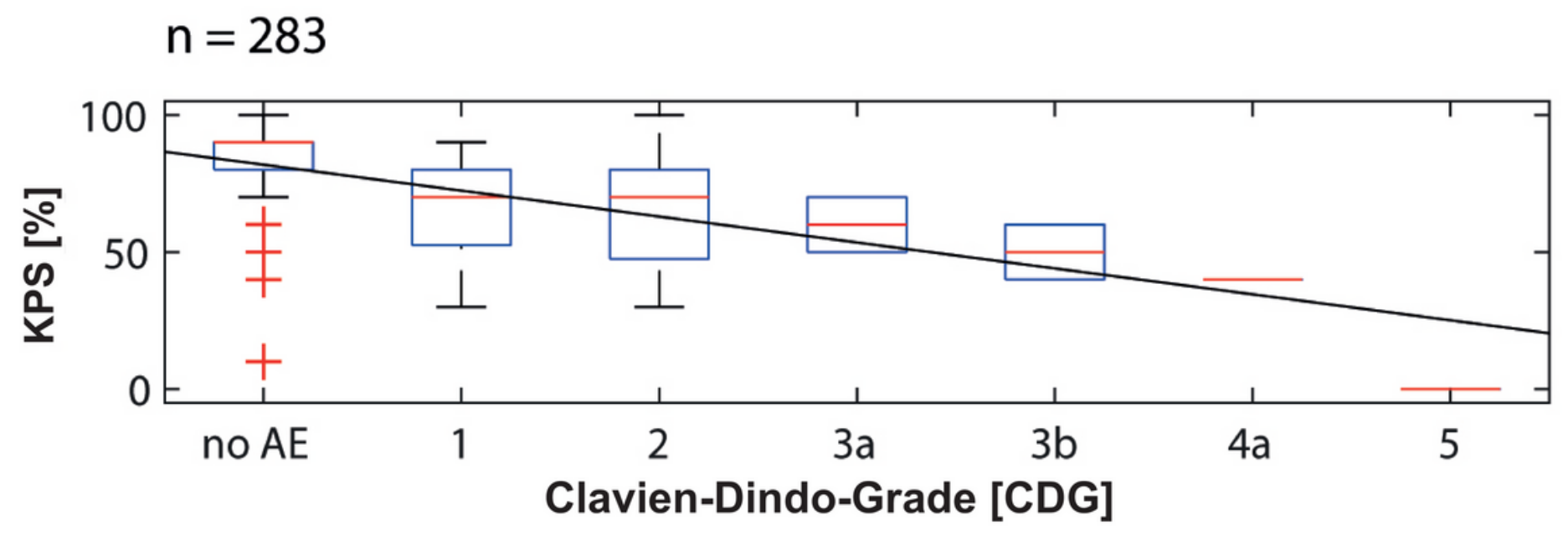

Figure 2

CDG grade and KPS. Over all 283 patients, in 78 patients at least one AE occurred before discharge. KPS and CDG at discharge were correlated with Spearman's rho $=-0.41(p<0.001)$. The linear fit had a slope of -9.5 KPS points per increment of CDG

\section{Supplementary Files}

This is a list of supplementary files associated with this preprint. Click to download.

- OnlineResource1.docx 